\title{
USING ELECTRIC CELL-SUBSTRATE IMPEDANCE SENSING TO MEASURE CHANGES IN THE PROJECTED AREA OF INDIVIDUAL CELLS
}

\author{
Pahnit Seriburi, Ashutosh Shastry, Tim Ren, \\ Steven Gales, Karl F. Böhringer and Deirdre Meldrum \\ Microscale Life Science Center, University of Washington, Seattle, WA, USA \\ (Tel: +1-206-616-2570; E-mail: pahnit@,u.washington.edu)
}

\begin{abstract}
We used an electrical cell-substrate impedance sensing (ECIS) technique to measure changes in the projected area of individual cells. The projected area of the cell plays a significant role in cellular behavior, including cell spreading, cell growth, cell death and cell adhesion. We built a custom impedance sensing setup; we recorded real time impedance spectra of individual adherent cells; and we used two parameters extracted from each impedance spectrum to measure the changes in the projected area of the cell. The projected area of the cell determines how much electrical current was measured using ECIS; therefore, it directly governed the values of two extracted impedance parameters.
\end{abstract}

Keywords: Impedance, cell-substrate, individual cells.

\section{INTRODUCTION}

The projected area of the cell plays a significant role in cellular behaviors. An increase in the projected area of individual cells with time determined the dynamic of cell spreading [1]. The final projected area of an individual cell after spreading influences several fundamental cellular behaviors of the cell such as cell growth, cell death, and cell adhesion [2-5].

Conventionally, a change in the projected area of the cell with time was measured by taking time series of optical images of a cell and processing the images with an analysis tool [1]. Recently, an alternative method based on ECIS developed by Giaever et al. [6,7] was proposed, but no supporting experimental data was presented [8]. Our preliminary results demonstrated that ECIS of individual adherent cells could measure the change in the projected area of individual cells. Experimental results would lead to a real-time, label-free, and non-invasive alternative method to study individual cell adhesion and cell spreading.

\section{MATERIALS AND METHODS}

We measured an impedance spectrum across an electrode pair when a cell was placed on a microelectrode (Fig. 1).

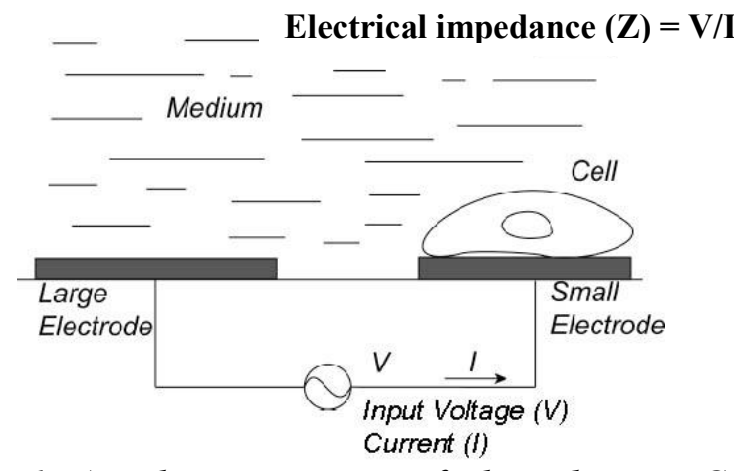

Fig. 1 A schematic view of the Electric Cellsubstrate Impedance Sensing (ECIS) technique for recording the projected area of individual cells. We seeded a cell on a small electrode (microelectrode) and obtained an impedance spectrum across an electrode pair that was submerged in cell medium.

We obtained microelectrodes (Applied Biophysics, Inc.) whose sizes are comparable to cellular dimensions and performed experiments using mouse macrophages (cell line: RAW264.7) (Fig. 2). Mouse macrophages are suitable cells for this experiment: adherent, autonomous, and simple to culture.

In this preliminary study, we followed individual mouse macrophages on a gold microelectrode both optically and electrically at $37^{\circ} \mathrm{C}$ and $5 \% \mathrm{CO}_{2}$. We divided the measurement period into four sub periods (T0-T3) using cell shape (round or not round) and the projected area of individual cells as criteria (Table 1). The 
projected cell areas were constant in the first two sub periods (T0 and T1) but the cell shape switched from round to not round after T1. In T2 sub period, the projected area of the cell increased steadily and reached its maximum at the end of T2. During the last sub period (T3), the projected area of the cell remained constant.

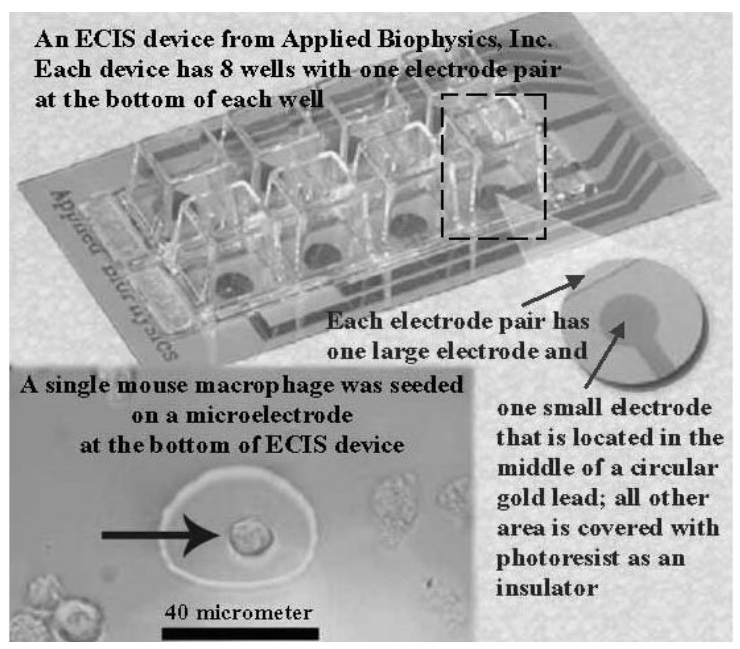

Fig. 2 A photograph of an ECIS device. Inset: An optical image of a macrophage on a microelectrode at the bottom of a well filled with DMEM cell medium. This optical image and the impedance spectrum $\left(Z_{\text {cell }}\right)$ were obtained simultaneously using ECIS and optical microscopy.

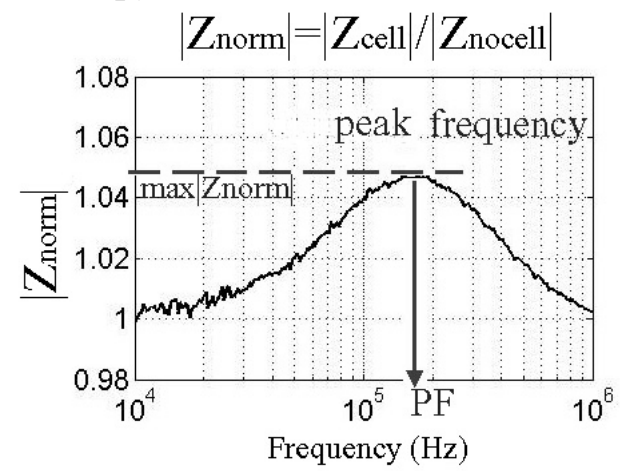

Fig. $3\left[Z_{\text {norm }} /\right.$ was obtained by dividing $\mid Z_{\text {cell }}$, the magnitude of an impedance spectrum of a microelectrode with a cell by $\mid Z_{\text {nocell }}$, the magnitude of an impedance spectrum of a microelectrode without a cell. Two parameters extracted from $Z_{\text {norm }} /$ were peak frequency (PF) and the maximum normalized magnitude of an impedance spectrum $\left(\max / Z_{\text {norm }}\right)$. Both values depended on a projected area and an average cell-substrate separation of individual cells.
Table 1 Individual macrophages on a gold microelectrode were followed over four sub periods (T0-T3). These sub periods were divided using cell shape and the projected area of the cell as criteria. 2D images of each cell were used to determine cell shape. The outline of each cell was defined by the user and the projected area of the cell was calculated using ImageJ software.

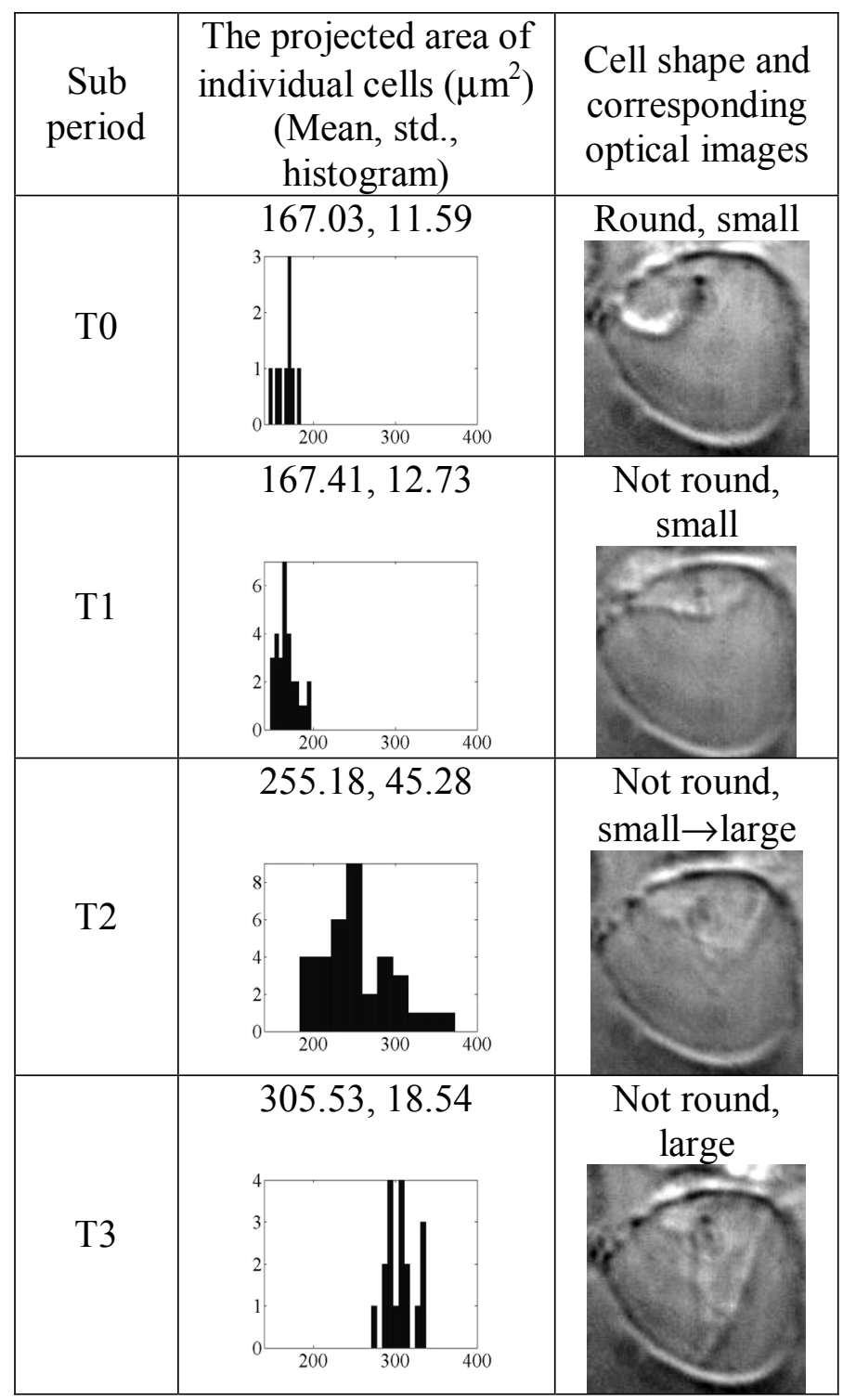

We measured the magnitude of an impedance spectrum of a microelectrode with a cell $\left(\left|Z_{\text {cell }}\right|\right)$ and the magnitude of an impedance spectrum of a microelectrode without a cell $\left(\left|Z_{\text {nocell }}\right|\right)$ using an impedance analyzer (Agilent 4294A). Simultaneously, we acquired an optical image of a microelectrode with or without a cell using a CCD camera (Lumenera LU075) equipped with a 40X 
objective (Edmund optics, DIN 40). We normalized $\left|Z_{\text {cell }}\right|$ using $\left|Z_{\text {nocell }}\right|$ to obtain $\left|Z_{\text {norm }}\right|$, the normalized magnitude of an impedance spectrum and extracted two parameters, the peak frequency (PF) and the maximum normalized magnitude of an impedance spectrum $\left(\max \left|Z_{\text {norm }}\right|\right)$, from the $\left|Z_{\text {norm }}\right|$ spectrum using MATLAB (Fig. 3). Their values depended on 1) the projected area of the cell and 2) the average cell-substrate separation of the cell [6-8]. The projected area of individual cells and their cell shapes were determined using ImageJ software.

\section{RESULTS AND DISCUSSION}

We compared the projected area of individual cells calculated from Image $J$, the $\max \left|\mathrm{Z}_{\text {norm }}\right|$ and the PF obtained from the ECIS measurement, and the corresponding optical images of individual cells taken from an optical microscopy in Fig. 4. Fig. $4 \mathrm{~b}$ showed an increase in $\max \left|\mathrm{Z}_{\text {norm }}\right|$ with time in $\mathrm{T} 2$ sub period while the $\max \left|\mathrm{Z}_{\text {norm }}\right|$ of other sub periods (T0, T1, T3) remained fairly constant. This corresponded very well with a change in the projected area of individual cells shown in Fig. 4a. Conversely, the PF changed rather quickly with time during T0, T1, and T3, but remained constant during T2 sub period (Fig. $4 c)$.

We plotted max $\left|Z_{\text {norm }}\right|$ against the projected area of individual cells and observed a monotonic relationship between these two parameters during T2 sub period (Fig. 5). However, PF remained constant when the projected area of the cell changed. This result suggested that $\max \left|\mathrm{Z}_{\text {norm }}\right|$ could be used to track changes in the projected area while PF could not.

This increase in $\max \left|Z_{\text {norm }}\right|$ during $T 2$ when there was an increase in the projected area of individual cells agreed with theoretical results $[7,8]$. Intuitively, when the projected area of a cell, i.e. the area of the microelectrode covered by the cell, increased, the current through the microelectrode was more limited, and we observed an increase of the magnitude of the impedance spectrum and an increase in $\max \left|Z_{\text {norm }}\right|$.

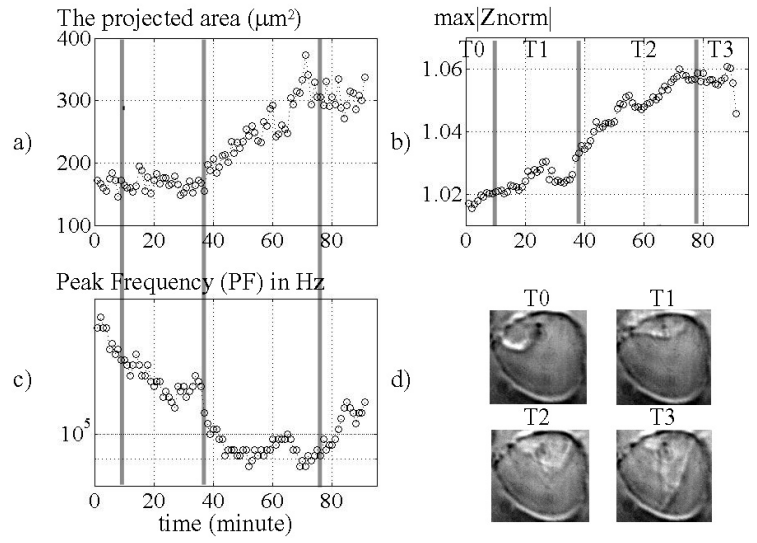

Fig. 4 a) A change in the projected area of individual cells with time (T0-T3 sub periods: 91 minutes total). Between T0 and T1 sub periods, the shape of the cell switched from round to not round while maintaining a constant area; b) $A$ change in $\max \left|Z_{\text {norm }}\right|$ with time for all sub periods. The increase in $\max \left|Z_{\text {norm }}\right|$ at T2 corresponded well with an increase in the projected area of the cell; c) A change in PF with time; d) Corresponding optical images of individual cells on a microelectrode during all sub periods (T0T3).

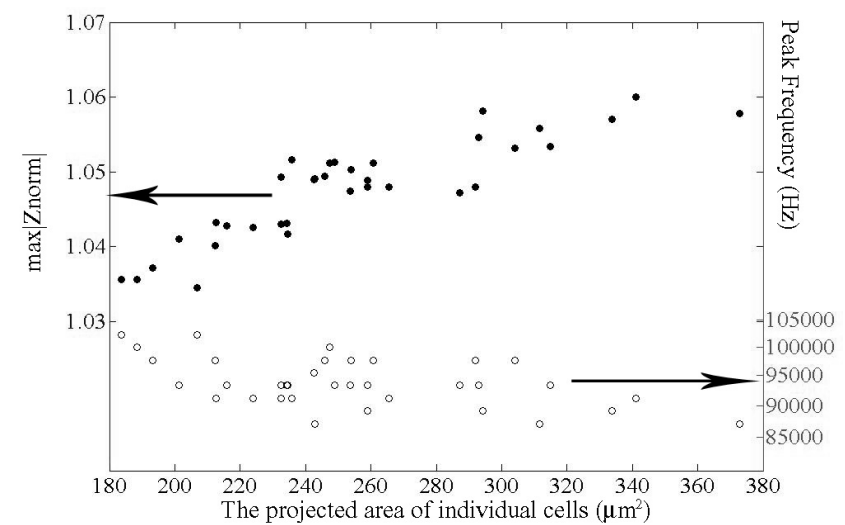

Fig. 5 The relationship between $\max \left|Z_{\text {norm }}\right|, P F$, and the projected area of individual cells during the T2 sub period. PF remained fairly constant during this period.

The PF was not sensitive to changes in the projected area of individual cells. Instead, it remained constant, which suggested that the average cell-substrate separation remained at a similar value during this $\mathrm{T} 2$ sub period [8]. 
Moreover, from Fig. 4b, we observed that $\max \left|Z_{\text {norm }}\right|$ increased slightly even though the projected area of individual cells remained constant during T0 and $\mathrm{T} 1$ sub periods. This was possible if the average cell-substrate separation decreased over time during these periods [8]. Indeed, this is likely since the PF, which directly relates to the average cell-substrate separation, decreased steadily during these sub periods [8] (Fig. 4c). Furthermore, during T3 sub period, we observed that $\max \left|Z_{\text {norm }}\right|$ decreased slightly even though the projected area of individual cells remained constant. Again, this is possible if the average cell-substrate separation increased over time during this period. In fact, we observed an increase in $\mathrm{PF}$ in this period, which implied a possible increase in the average cell-substrate separation. Thus, these preliminary results suggested that there was a reciprocal relationship between two extracted impedance parameters, $\max \left|Z_{\text {norm }}\right|$ and $P F$, and both parameters were linked by one of the properties of the cell, that is the average cell-substrate separation.

Although our results suggested that the average cell-substrate separation remained constant in T2 sub period and changed with time in other sub periods (T0, T1, T3), we did not have other independent means to confirm this observation. A measurement of the average cell-substrate separation of individual cells in real time using optical means [9-10] is underway. This will allow us to correlate both $\max \left|Z_{\text {norm }}\right|$ and $P F$ to the average cell-substrate separation. Consequently, we will be able to use ECIS to follow both the projected area as well as the cell-substrate separation of individual cells in real time [11]. Eventually, we plan to use these two properties of individual cells as two metrics of cell spreading and cell adhesion.

\section{CONCLUSION}

Our preliminary results show that only $\max \left|Z_{\text {norm }}\right|$ is sensitive to the projected area of individual cells and there is a reciprocal relationship between two extracted impedance parameters, $\max \left|Z_{\text {norm }}\right|$ and $P F$. We speculate that $P F$ and $\max \left|Z_{\text {norm }}\right|$ are linked by one of the properties of the cell: the cell-substrate separation. This work marks a step towards a real time measurement of two properties of individual cells: the projected area and the average cell-substrate separation, and more experiments are underway.

\section{ACKNOWLEDGEMENT}

We gratefully acknowledge the support from the NIH National Human Genome Research Institute, Centers of Excellence in Genomic Science, Grant Number 1 P50 HG002360-01, CEGSTech: Integrated Biologically-Active Microsystems and NIH NHGRI Grant Number 1 R01 HG01497-06: Microscale Instrument Development for Genomic Analysis.

\section{REFERENCES}

[1] Itano, N., Okamoto, S., Zhang, D, Lipton, S. A. \& Ruoslahi, E. (2003) PNAS 100, 5181-5186 [2] Chen, C. S., Alonso, J. L., Ostuni, E., Whitesides, G. M. \& Ingber, D. E. (2003) Biochemical and Biophysical Research Communications 307, 355-361

[3] Gallent, N. D., Michael, K. E. \& Garcia, A. J. (2005) Molecular Biology of the Cell 16, 43294340

[4] Lotz, M. M., Burdsal, C. A., Erickson, H. P. \& McClay, D. R. (1989) The Journal of Cell Biology 109, 1795-1805

[5] Chen, C. S., Mrksich, M., Huang, S., Whitesides, G. M. \& Ingber, D. E. (1997) Science 276, 1425-1428

[6] Giaever, I. \& Keese, C. R. (1993) Nature 366, 591-592

[7] Wegener, J., Keese, C. R. \& Giaever, I. (2000) Experimental Cell Research 259, 158-166

[8] Huang, X., Nguyen, D., Greve, D. W. \& Domach, M. M. (2004) IEEE Sensors Journal 4, 576-583

[9] Llobet, A., Beaumont, V. \& Lagnado, L. (2003) Neuron 40, 1075-1086

[10] Braun, D. \& Fromherz, P. (1997) Applied Physics A 65, 341-348

[11] Lidstrom, M. \& Meldrum, D. (2003) Nature

Reviews Microbiology 1, 158-164 